\title{
CHIANTI - An atomic database for emission lines. Version 8
}

\author{
G. Del Zanna ${ }^{1}$, K. P. Dere ${ }^{2}$, P. R. Young ${ }^{3}$, E. Landi ${ }^{4}$, and H. E. Mason ${ }^{1}$ \\ 1 DAMTP, Centre for Mathematical Sciences, University of Cambridge, Wilberforce road, Cambridge, CB3 0WA, UK \\ e-mail: g.del-zanna@damtp.cam.ac.uk \\ 2 School of Physics, Astronomy and Computational Sciences, MS 6A2, George Mason University, 4400 University Drive, Fairfax, \\ VA 22030, USA \\ 3 College of Science, George Mason University, 4400 University Drive, Fairfax, VA 22030, USA \\ ${ }^{4}$ Department of Climate and Space Sciences and Engineering, University of Michigan, Ann Arbor, MI 48109, USA
}

Received 24 June 2015 / Accepted 12 August 2015

\section{ABSTRACT}

\begin{abstract}
We present version 8 of the CHIANTI database. This version includes a large amount of new data and ions, which represent a significant improvement in the soft X-ray, extreme UV (EUV) and UV spectral regions, which several space missions currently cover. New data for neutrals and low charge states are also added. The data are assessed, but to improve the modelling of low-temperature plasma the effective collision strengths for most of the new datasets are not spline-fitted as previously, but are retained as calculated. This required a change of the format of the CHIANTI electron excitation files. The format of the energy files has also been changed. Excitation rates between all the levels are retained for most of the new datasets, so the data can in principle be used to model highdensity plasma. In addition, the method for computing the differential emission measure used in the CHIANTI software has been changed.
\end{abstract}

Key words. atomic data - line: identification - atomic processes - radiation mechanisms: thermal

\section{Introduction}

CHIANTI $^{1}$ is a database of assessed atomic parameters and transition rates needed for the calculation of the line and continuum emission of optically thin, collisionally-dominated plasma. IDL-based software was initially developed to calculate synthetic spectra and measure plasma parameters such as electron densities and temperatures, and is distributed within Solarsoft ${ }^{2}$. A Python version of the CHIANTI software is also available ${ }^{3}$.

CHIANTI was first released in 1996 (Dere et al. 1997) and since then various new releases have been made available, to expand the database and improve the quality of the data. Particular emphasis has been given to the line identifications and improvement of the reference wavelengths. For several ions, the wavelengths and identifications are different from and more accurate than those of the NIST database (Kramida et al. 2013), as recently confirmed by laboratory measurements (Beiersdorfer \& Lepson 2012; Beiersdorfer et al. 2014). For the above reasons, CHIANTI is now often used as a reference atomic database for ions, and is included in several other atomic codes and packages.

The version 8 of the database focuses on the inclusion of a large amount of atomic data, much of which has been produced by the UK APAP network ${ }^{4}$. The new data are particularly relevant for current EUV/UV spectroscopic instruments aboard the Solar and Heliospheric Observatory (SOHO), Hinode, Solar Dynamics Observatory (SDO), and Interface Region Imaging Spectrometer (IRIS) satellites. They are also important for

\footnotetext{
http://www . chiantidatabase.org

http://www. lmsal.com/solarsoft/

chiantipy. sourceforge.net/

http : //www . apap-network . org
}

the X-rays (especially soft X-rays), observed by Chandra and XMM-Newton. Version 8 also adds data for low charge states that were missing and are relevant for modelling the UV spectral region.

Section 2 describes the database improvements. Section 3 describes the new CHIANTI DEM software and shows as an example how the new data improves the analysis of SDO data. Section 4 provides the conclusions.

\section{Database improvements}

\subsection{New database format}

The formats of the core energy level (elvlc), radiative (wgfa) and electron collision (splups) data files of CHIANTI have remained unchanged since version 1 (Dere et al. 1997), except for a modification to the splups files in version 4 (Young et al. 2003) to allow 9-point spline fits to the data in addition to the 5-point spline fits. The files are in ascii format, with a fixed text width for each column and, in preparation for the inclusion of atomic models with $\geq 1000$ atomic levels, it has been necessary to modify the format of the elvlc and splups files, which were restricted to at most 999 levels. The wgfa files retain the same structure as in previous versions of the database. The elvlc files have been restructured (Appendix A.1) but retain the same name, while the splups files have been significantly revised (Appendix A.2) and are now renamed as "scups" files. For both the updated elvlc and new scups files, an ascii fixed text width format has been retained.

A fundamental change in philosophy for the electron collision data has been implemented through the new scups files. Previously effective collision strengths (either directly taken 
from the literature or integrated from collision strengths) were scaled using the Burgess \& Tully (1992) method onto a scaled temperature $T_{\mathrm{s}}$ domain between 0 and 1 , then either a five or nine point spline was fit to the data in the scaled domain. The five or nine point spline nodes were stored in the splups files. For version 8 we continue to scale the effective collision strength data, but now we directly store these scaled data points and no longer perform the five or nine point spline fits. An extrapolation to $T_{\mathrm{s}}=0$ is performed, and either the high temperature limit point (Dere et al. 1997) or an extrapolation is used for the $T_{\mathrm{s}}=1$ value. This ensures that an effective collision strength can be derived for any temperature between zero and infinity through interpolation of the stored data points.

The advantage of the new format is that the stored CHIANTI data is now a direct representation of the original data and there is no need to omit data points as was sometimes necessary if the collision strength structure could not be represented accurately by a nine point spline fit. This often occurred at low temperatures. Providing the actual rates represents an improvement in accuracy for studies of very low temperature plasma, such as those of photoionised plasma. We continue to assess the collision data using a mixture of automatic checking procedures and by-eye inspection.

Much of the new atomic data added to version 8 has been processed in this way. For data added in earlier versions of the database and retained for version 8 we have simply re-formatted the previous file formats. For example, if a transition was represented by a nine point spline fit, then these nine spline points are stored in the new scups file.

A further advantage of the new method is that it is much more efficient to process a data-set, allowing the complete set of collision strengths for an ion to be added. This is in contrast to earlier versions of CHIANTI for which often only transitions involving a metastable level were included. This advance will allow the CHIANTI atomic models to be used in a high-density regime as found in, for example, laboratory plasmas. When calculating the level populations, the rates are obtained, as before, by interpolation in the scaled domain.

\subsection{He-like ions: $C V, N V I, O V I I, N e I X, M g X I, A I X I I, S i X I I I$, $S X V$, ArXVII, CaXIX, Fe XXV, NiXXVII, Zn XXIX}

For the He-like ions the main changes have been to insert the UK APAP network radiation-damped $R$-matrix effective collision strength calculations of Whiteford et al. (2001) into the database for the ions listed. Collision strengths are provided between all levels of a 49-level fine structure model that includes all levels up through $1 \mathrm{~s} 51$. These new collision strengths replace those in the 7.1 version of the CHIANTI database (Landi et al. 2013). In the cases of C V, O VII, Mg XI, Al XII, Si XIII, Ni XXVII, the previous collision strengths were taken from the distortedwave calculation of Zhang \& Sampson (1987) who provided collision strengths between the $1 \mathrm{~s}^{2}, 1 \mathrm{~s} 2 \mathrm{~s}$, and $1 \mathrm{~s} 2 \mathrm{p}$ fine-structure levels. Collision strengths to $n=3-5$ were taken from the calculations of Sampson et al. (1983) which were performed with a hydrogenic approximation. The new data therefore represent an improvement, especially for the forbidden transitions, which are increased by the effect of the resonances (although at high temperatures increases are only of the order of 10\%).

For SXV and CaXIX, the previous version of CHIANTI used the $R$-matrix calculations of Kimura et al. (2000) that provided collision strengths between the $1 \mathrm{~s}^{2}, 1 \mathrm{~s} 21$ and $1 \mathrm{~s} 31$ levels. Excitation to the $1 \mathrm{~s} 41$ and $1 \mathrm{~s} 51$ were the hydrogenic collision strengths of Sampson et al. (1983). For N VI, the version 7.1 collision strengths were taken from the combined $R$-matrix and FAC (Flexible Atomic Code, see Gu 2008) calculations of Aggarwal et al. (2009). These calculation are of similar accuracy to the APAP calculations but the latter have been inserted into the current database for consistency.

For ArXVII and Fe XXV, version 7.1 already included the rates of Whiteford et al. (2001). Zn XXIX is new to the CHIANTI database.

The version 7.1 energy level files, e.g. fe_25.elvlc have been updated. The basic theoretical energies have been obtained with AUTOSTRUCTURE (Badnell 2011) calculations. These are used to provide level energies and to provide approximate wavelengths in cases where energies based on observed spectral lines are unavailable. Many of the observed energies in previous versions have been taken from the NIST database (Kramida et al. 2013) and these have been updated to the latest version (5.1) of the NIST database. Highly accurate calculations of the energies of the six $1 \mathrm{~s} 2 \mathrm{~s}$ and $1 \mathrm{~s} 2 \mathrm{p}$ fine structure levels provided by Artemyev et al. (2005) have been included.

The updated energies have been used to calculate the wavelengths in the radiative data files, e.g. fe_25.wgfa. Weighted oscillator strengths and A-values are obtained from an AUTOSTRUCTURE calculation. A-values originating from a number of the 1s21 levels, varying from ion to ion, have been retained from version 7.1.

The effect of cascades following recombination into He-like levels of C V, N VI, O VII, Ne IX, Mg XI and Si XIII was modeled by Porquet \& Dubau (2000), and these data were added to CHIANTI v.6 (Dere et al. 2009). Porquet \& Dubau (2000) provided total rates into the individual $1 \mathrm{~s} 21$ levels for six temperatures covering $1.6 \mathrm{dex}$ in log temperature. One problem with this data-set is that the CHIANTI models for these ions contain 49 levels and the cascading through levels 8-49 which would produce emission lines is not modeled. In particular the lines due to dielectronic recombination that are important at $\mathrm{X}$-ray wavelengths are not modeled and so it was necessary to retain the dielectronic files for these ions (see Dere et al. 2001). This has the consequence that emission lines from the $1 \mathrm{~s} 21$ levels (with indices 2-7 in the CHIANTI model) had additional contributions due to double-counting of the recombination cascades.

In addition, the temperature coverage of the Porquet \& Dubau (2000) recombination rates was small, and not suitable for the wide range of uses of the CHIANTI database. For these reasons, the Porquet \& Dubau recombination rates have been replaced with the level-resolved radiative recombination rates of Badnell (2006) into all 49 levels of the CHIANTI model. The level-resolved dielectronic recombination rates contained in the dielectronic models of the ions have been retained from the earlier versions of the database. As the Badnell (2006) data are direct rates into individual levels, then the new CHIANTI model does not include contributions from levels with $n>5$ in contrast to Porquet \& Dubau (2000) who included cascading from all values of $n$. These additional contributions however are small.

For the remaining He-like ions, the previous CHIANTI models used radiative recombination rates from Mewe et al. (1985) computed using the prescription of Mewe \& Gronenschild (1981). These rates are available for the $1 \mathrm{~s} 21$ levels and selected other levels, and they include the contributions from cascading. They have been replaced with the direct recombination rates of Badnell (2006) into all levels of the CHIANTI models. The level-resolved dielectronic rates from the previous CHIANTI models that are stored in the dielectronic files are retained. 
To reduce the size of the data files containing the radiative rates, we have retained for all the new datasets only transitions with a branching ratio greater than $10^{-5}$.

\subsection{Li-like ions}

Liang \& Badnell (2011) have performed, within the UK APAP network, scattering calculations for electron-impact excitation of all Li-like ions from $\mathrm{Be}^{+}$to $\mathrm{Kr}^{33+}$ using the radiationand Auger-damped intermediate-coupling frame transformation $R$-matrix approach. The target included 204 close-coupling (CC) levels, with valence (up to $n=5$ ), and core-electron excitations (up to $n=4$ ). During the assessment of these datasets, we have uncovered small inconsistencies in a few ions between the highest temperature values of the published rates and their high-temperature limits. These were ultimately due to a mistaken repetition of the last few collision strengths. The data have been corrected and the effective collision strengths recalculated (Liang 2013, priv. comm.). The targets represent a considerable improvement over previous ones, which typically contained a much smaller number of levels, either 15 or 40.

\subsubsection{CIV, N V, O VI, Ne VIII, MgX, AIXI, SiXII, SXIV, ArXVI, CaXVIII, CrXXII, FeXXIV, NiXXVI, Zn XXVIII}

The basic ion structure is determined from an AUTOSTRUCTURE calculation that provides theoretical energy levels, oscillator strengths, A-values and autoionization rates. However, the energies, wavelengths and autoionization rates are taken from the calculations of Safronova (as reported in Kato et al. 1997 or privately communicated), where available. As with the abundant He-like ions, we have used the direct radiative recombination rates of Badnell (2006) to account for the effect of radiative recombination on the level populations. We have used AUTOSTRUCTURE to calculate the bound levels up to $n=8$ for which the direct recombination rates are available.

The $n=6,7,8$ levels that have been added do not have any direct collisional excitation, so they are only populated through recombination. This means that for normal collisionally-excited plasma the intensities of any lines produced by these levels are significantly underestimated, and should therefore not be used for spectral analysis.

We have included only the collision strengths of Liang \& Badnell (2011) from the $2 \mathrm{~s}$ and 2 p levels. The data have been spline-fitted over a reduced number of temperatures.

A large number of the observed energy levels have been taken from Kramida et al. (2013). However, we found that for several important ions the NIST energies were in need of improvement. Details on each ion are provided as comments within the energy files.

For O VI, several wavelengths have been taken from experimental measurements and are shown in Table 1. These wavelengths have also been used to provide the "observed" energies for their respective upper levels.

For Ne VIII, the wavelength of the $1 s^{2} 2 s^{2} \mathrm{~S}_{1 / 2}-1 \mathrm{~s}^{2} 2 \mathrm{p}^{2} \mathrm{P}_{3 / 2}$ transition has been measured by Peter \& Judge (1999) and Dammasch et al. (1999). These measurements are consistent with each other and have been used to determine the energy of the $1 s^{2} 2 p^{2} \mathrm{P}_{3 / 2}$ level and have been used as the "observed" wavelength.

Peter \& Judge (1999) have also measured the wavelength of the $\mathrm{MgX} 1 \mathrm{~s}^{2} 2 \mathrm{~s}{ }^{2} \mathrm{~S}_{1 / 2}-1 \mathrm{~s}^{2} 2 \mathrm{p}{ }^{2} \mathrm{P}_{1 / 2}$ line. This measurement has
Table 1. Experimental wavelengths for O VI.

\begin{tabular}{llll}
\hline \hline$\lambda(\AA)$ & Lower & Upper & Ref. \\
\hline 23.017 & $1 \mathrm{~s}^{2} 2 \mathrm{p}^{2} \mathrm{P}_{1 / 2,3 / 2}$ & $1 \mathrm{~s} 2 \mathrm{~s}^{2}{ }^{2} \mathrm{~S}_{1 / 2}$ & Gu et al. (2005) \\
22.374 & $1 \mathrm{~s}^{2} 2 \mathrm{~s}^{2} \mathrm{~S}_{1 / 2}$ & $1 \mathrm{~s} 2 \mathrm{~s}\left({ }^{3} \mathrm{~S}\right) 2 p{ }^{4} \mathrm{P}_{1 / 2}$ & Gu et al. (2005) \\
22.019 & $1 \mathrm{~s}^{2} 2 \mathrm{~s}^{2} \mathrm{~S}_{1 / 2}$ & $1 \mathrm{~s} 2 \mathrm{~s}\left({ }^{1} \mathrm{~S}\right) 2 p{ }^{2} \mathrm{P}_{1 / 2,3 / 2}$ & Schmidt et al. (2004) \\
21.845 & $1 \mathrm{~s}^{2} 3 \mathrm{~s}^{2} \mathrm{~S}_{1 / 2}$ & $1 \mathrm{~s} 2 \mathrm{~s}\left({ }^{1} \mathrm{~S}\right) 3 p{ }^{2} \mathrm{P}_{1 / 2}$ & Gu et al. (2005) \\
21.672 & $1 \mathrm{~s}^{2} 3 \mathrm{~s}^{2} \mathrm{~S}_{1 / 2}$ & $1 \mathrm{~s} 2 \mathrm{~s}\left({ }^{3} \mathrm{~S}\right) 3 d^{4} \mathrm{P}_{3 / 2}$ & Gu et al. (2005) \\
\hline
\end{tabular}

been used to derive the "observed" energy of the $1 s^{2} 2 p^{2} \mathrm{P}_{1 / 2}$ level and the "observed" wavelength of that line in CHIANTI.

For Fe XXIV, observed energies for the ground level through $1 s^{2} 5 d$ have been taken from Del Zanna (2006).

In the version 7 of the CHIANTI database, the atomic models for these ions were assembled largely between 1997 and 2001. The model for Fe XXIV was assembled in 2005. The database for these ions reflect the atomic data that was available at the time. Energy levels were taken from version 2 of the NIST database and from the series of NIST publications such as Martin et al. (1990) for the sulphur ions. A-values were taken from Martin et al. (1993) or derived from the oscillator strengths provided in the collision strength calculations. Autoionization rates were taken from Vainshtein \& Safronova (1978) that were updated by U. Safronova in 1999. Collision strengths were taken from a variety of sources. For a few ions, distorted-wave collision strengths calculated by Zhang et al. (1990) for the bound levels up to $5 \mathrm{~g}$ were included, together with inner-shell excitation rates provided by Goett \& Sampson (1983). C IV and N V only included 15 levels, up to $4 \mathrm{f}$. The data provided in version 8 therefore marks a considerable improvement over the previous version.

To reduce the size of the data files containing the radiative rates, we have retained for all the new datasets only transitions with a branching ratio greater than $10^{-5}$.

\subsubsection{Na IX, PXIII, CIXV, KXVII, MnXXIII, and CoXXV}

For these ions we have retained the original 204 levels and the original energies, A-values and all the effective collision strengths as calculated by Liang \& Badnell (2011). To reduce the size of the data files containing the radiative rates, we have retained for all the new datasets only transitions with an A-value greater than $10^{-5}$ the largest A-value (for each level).

We have calculated the autoionization rates using AUTOSTRUCTURE (version 24.24) and the same set of scaling parameters used by Liang \& Badnell (2011) for consistency. For NaIX, experimental energies have been reassessed on the basis of the wavelength measurements as reported in Kelly (1987). For P XIII, Cl XV, K XVII, Mn XXIII, and Co XXV experimental energies have been taken from Kramida et al. (2013).

In the version 7 of the CHIANTI database, Na IX, P XIII, K XVII, Mn XXIII, and Co XXV included only 20 levels up to 5d, and DW collision strengths were provided by Zhang et al. (1990) $\mathrm{Cl} \mathrm{XV}$ is a new entry in the database.

\subsection{B-like ions}

Liang et al. (2012) have performed, within the APAP network, ICFT $R$-matrix calculations for electron-impact excitation amongst 204 close-coupling levels for all boron-like ions from $\mathrm{C}^{+}$to $\mathrm{Kr}^{31+}$. We include the Liang et al. (2012) collision 
strengths and A-values for several ions, as described below. We note, however, that we have replaced the A-values of the transitions among the lowest levels as calculated by Liang et al. (2012) with AUTOSTRUCTURE with more accurate values, whenever available. For example, those calculated by Corrégé \& Hibbert (2004) with the CIV3 code (Hibbert 1975) and those calculated by Rynkun et al. (2012) with the multi-configuration DiracHartree-Fock (MCDHF) GRASP2K code (Jönsson et al. 2007). This is because the ab initio energies of the CIV3 and GRASP2K are normally more accurate than those by AUTOSTRUCTURE, and so are the A-values.

The Fe XXII ion model included the Badnell et al. (2001) collision strengths so it is left unchanged, since the Liang et al. (2012) calculation is essentially the same.

During the present assessment, we have uncovered an error in the data. The $2 \mathrm{~s} 2 \mathrm{p}^{2}{ }^{2} \mathrm{~S}_{1 / 2},{ }^{2} \mathrm{P}_{1 / 2}$ levels (Nos. 8, 9) were inverted by mistake, hence the collision strengths and A-values for transitions connected to these levels were incorrect. The data for these transitions have been recalculated (Liang, priv. comm.). To reduce the size of the data files containing the radiative rates, we have retained for all the new datasets only transitions with an A-value greater than $10^{-5}$ the largest A-value (for each level).

\subsubsection{II}

C II produces a number of strong emission lines in the UV, including the $2 \mathrm{~s}^{2} 2 \mathrm{p}^{2} \mathrm{P}_{J}-2 \mathrm{~s} 2 \mathrm{p}^{2}{ }^{2} \mathrm{D}_{J^{\prime}}$ multiplet found between 1334.5 and $1335.7 \AA$ that is routinely observed by the recentlylaunched IRIS instrument (De Pontieu et al. 2014). For C II, the collisional data of Liang et al. (2012) replace those of Tayal (2008), which included only 37 levels. Observed energies are taken from NIST (Kramida et al. 2013). A-values are taken from Liang et al. (2012), with the exception of several transitions of the lower levels, where the Corrégé \& Hibbert (2004) data are used. We note that relatively good agreement is found between these A-values and those of Liang et al. (2012), but not with those of Tayal (2008).

\subsubsection{N III}

For N III, the Liang et al. (2012) collisional data replace those of Stafford et al. (1994) which included only 20 levels. This is a significant improvement. A-values are taken from Liang et al. (2012), with the exception of the transitions of the lower 20 levels $\left(2 \mathrm{~s} 2 \mathrm{p}^{2}, 2 \mathrm{p}^{3}, 2 \mathrm{~s}^{2} 31\right)$ calculated by Corrégé \& Hibbert (2004), which were already present in the previous version of CHIANTI. Observed energies have been taken from NIST (Kramida et al. 2013).

\subsubsection{O IV}

O IV is an important ion for solar physics applications, in particular for measuring electron densities. The Liang et al. (2012) collisional data for O IV replace those of Aggarwal \& Keenan (2008) which included only 75 levels.

There is overall agreement between the two calculations, with differences of the order of $10 \%$ for the important transitions in the IRIS wavelength range (see Dudík et al. 2014).

Observed energies have been taken from the previous CHIANTI version and from NIST (Kramida et al. 2013), except those of the $2 \mathrm{~s} 2 \mathrm{p}^{2}$ levels, which have been revised. A-values are taken from Liang et al. (2012), with the exception of the transitions among the lower 20 levels $\left(2 \mathrm{~s} 2 \mathrm{p}^{2}, 2 \mathrm{p}^{3}, 2 \mathrm{~s}^{2} 31\right)$ calculated by Corrégé \& Hibbert (2004), which were already present in the previous version of CHIANTI.

\subsubsection{Ne VI}

The Liang et al. (2012) collisional data replace those from a similar calculation, an 180-level intermediate-coupling frametransformation (ICFT) $R$-matrix close-coupling calculation by Mitnik et al. (2001). Observed energies have been taken from NIST (Kramida et al. 2013). A-values are taken from Liang et al. (2012) with the exception of the transitions among the lower 15 levels $\left(2 \mathrm{~s} 2 \mathrm{p}^{2}, 2 \mathrm{p}^{3}\right)$ for which we have adopted the results of the MCDHF calculations obtained by Rynkun et al. (2012) with the GRASP2K code.

\subsubsection{Mg VIII}

For Mg VIII, we have adopted the $R$-matrix ICFT collisional data of Liang et al. (2012). The new collisional data replace the DW data of Zhang \& Sampson (1994), which only contained excitation within the $15 n=2$ levels, and unpublished calculations from Sampson \& Zhang (1995) for the $n=3$ levels. Observed energies have been taken from NIST (Kramida et al. 2013). A-values are taken from Liang et al. (2012) with the exception of the transitions among the lower 15 levels $\left(2 \mathrm{~s} 2 \mathrm{p}^{2}, 2 \mathrm{p}^{3}\right)$ for which we have adopted the results of the MCDHF calculations obtained by Rynkun et al. (2012) with the GRASP2K code.

\subsubsection{SiX}

SiX is a particularly important ion for measuring electron densities of the solar corona (see e.g. an example in Del Zanna 2012b). The Liang et al. (2012) data replace the Liang et al. (2009a) ICFT $R$-matrix calculation, which included 125 fine-structure levels (58 LS terms) belonging to the spectroscopically-important $n=2,3$ configurations. The experimental energies for this ion were assessed for version 7 (Landi et al. 2012), and have been taken from a variety of sources. A-values are taken from Liang et al. (2012) with the exception of the transitions among the lower 15 levels $\left(2 \mathrm{~s} 2 \mathrm{p}^{2}, 2 \mathrm{p}^{3}\right)$ for which we have adopted the results of the MCDHF calculations obtained by Rynkun et al. (2012) with the GRASP2K code.

\subsubsection{AlIX, SXII, ArXIV, and CaXVI}

Al IX, S XII, Ar XIV, and Ca XVI are ions that are particularly important for solar physics diagnostics. For example, S XII, Ar XIV, and Ca XVI produce lines in the EUV observed with the Hinode EUV Imaging Spectrograph (EIS), and that have recently been used for elemental abundance studies (cf. Del Zanna 2013).

The ion models for AlIX, S XII, ArXIV, and CaXVI, as described in version 1 (Dere et al. 1997), contained several datasets, with some unpublished data. In terms of collision strengths, they included $R$-matrix calculations of Zhang et al. (1994) for the 15 fine-structure levels arising from the $n=$ 2 configurations. Collision strengths to the 110 fine-structure $n=3$ levels were unpublished, taken from a simple CoulombBorn calculation by Zhang \& Sampson (1995). An error in the $R$-matrix calculations of Zhang et al. (1994) affecting Al IX, Si X, S XII ArXIV, and CaXVI was uncovered, and corrected rates were introduced in version 4 (Young et al. 2003).

We have replaced the previous collision rates with the Liang et al. (2012) APAP data. As noted by Liang et al. (2012), the 
new data have significantly larger collision strengths for the $n=$ 2 levels, due to the extra resonances attached to the $n=3$ levels, and which were not included in the Zhang et al. (1994) calculations. As shown in Fig. 4 of Liang et al. (2012), for Ar XIV there is good agreement (within a relative 20\%) only for the strongest transitions; however, the effect of the resonances increases the collision strengths by about a factor of two or more for the weaker transitions.

Observed energies have been taken mostly from NIST (Kramida et al. 2013), although we note that in several cases values are only known relative to the energies of other levels. A-values are taken from Liang et al. (2012) with the exception of the transitions among the lower 15 levels $\left(2 \mathrm{~s} 2 \mathrm{p}^{2}, 2 \mathrm{p}^{3}\right)$ for which we have adopted the results of the MCDHF calculations obtained by Rynkun et al. (2012) with the GRASP2K code.

For AlIX, Ar XIV, and CaXVI we used the corrected collisional data involving the two levels $(8,9)$.

For S XII, the intensities of the strongest EUV lines are very similar to those of the previous model. The energies of some S XII levels are taken from Lepson et al. (2005).

\subsubsection{Na VII, PXI, KXV, CrXX, MnXXI, CoXXIII}

For Na VII, P XI, K XV, CrXX, MnXXI and Co XXIII the Liang et al. (2012) collisional data replace the DW data of Zhang \& Sampson (1994), which only contained excitation within the $15 n=2$ levels. Observed energies have been taken from NIST (Kramida et al. 2013), although we note that in several cases values are only known relative to the energies of other levels.

For Na VII, P XI, K XV we used the corrected collisional data involving the two levels $(8,9)$.

Observed energies have been taken from NIST (Kramida et al. 2013). A-values are taken from Liang et al. (2012) with the exception of the transitions among the lower 15 levels $\left(2 \mathrm{~s} 2 \mathrm{p}^{2}\right.$, $2 \mathrm{p}^{3}$ ) for which we have adopted the results of the MCDHF calculations obtained by Rynkun et al. (2012) with the GRASP2K code.

\subsubsection{CIXIII, NiXXIV}

$\mathrm{Cl}$ XIII is a new entry to the database. We used the collision rates of Liang et al. (2012), with the corrected data involving the two levels $(8,9)$. Observed energies have been taken from NIST (Kramida et al. 2013). A-values are taken from Liang et al. (2012) with the exception of the transitions among the lower 15 levels $\left(2 \mathrm{~s} 2 \mathrm{p}^{2}, 2 \mathrm{p}^{3}\right)$ for which we have adopted the results of the MCDHF calculations obtained by Rynkun et al. (2012) with the GRASP2K code.

For Ni XXIV, the Liang et al. (2012) collisional data replace those calculated by Sampson et al. (1986). Observed energies have been taken from NIST (Kramida et al. 2013). A-values are taken from Liang et al. (2012) with the exception of the transitions among the lower 15 levels $\left(2 \mathrm{~s} 2 \mathrm{p}^{2}, 2 \mathrm{p}^{3}\right)$ for which we have adopted the results of the MCDHF calculations obtained by Rynkun et al. (2012) with the GRASP2K code.

\subsection{C-like ions}

\subsubsection{Cl}

C $\mathrm{I}$ is a new addition to the database. Wang et al. (2013) have recently performed a $B$-spline $R$-matrix calculation for electronimpact excitation and ionization of carbon. Wang et al. (2013) provided $L S$-resolved effective collision strengths, but only covering high temperatures (above $10000 \mathrm{~K}$ ). One of the authors (O. Zatsarinny) has recalculated the LSJ-resolved effective collision strengths over a more extended temperature range (from 1000 to $500000 \mathrm{~K}$ ), and also provided us with the level-resolved oscillator strengths and A-values for the E1, E2 and M2 transitions, which we have included. We have verified that the effective collision strengths converge to the high-energy limits for the allowed transitions.

A-values for the forbidden lines within the ground configuration are taken from the CIV3 calculations of Hibbert et al. (1993). Experimental energies have been taken from Chang \& Geller (1998).

\subsection{N-like ions}

\subsubsection{NI}

We have included new effective collision strengths and gf-values for N I from Tayal (2006). The collision strengths were obtained with the $R$-matrix B-spline method, including 24 spectroscopic bound and autoionising states together with 15 pseudostates. This calculation is much larger than the 18-states previous one from Tayal (2000), which was added in CHIANTI version 4 (Young et al. 2003).

A-values for the allowed and forbidden lines have been taken from Tachiev \& Froese Fischer (2002). They were calculated with the energy-adjusted MCHF method. Observed energies have been taken from NIST (Kramida et al. 2013).

\subsection{O-like ions}

\subsubsection{FeXIX}

For Fe XIX we have included the collision strengths from the $R$-matrix calculations of Butler \& Badnell (2008). The target included 342 close-coupling levels up to $n=4$. They replace those calculated with the DW approximation and the FAC code by $\mathrm{Gu}$ (2003). We have retained the extra levels (from 343 to 636) of the previous CHIANTI model, which included data from Landi \& $\mathrm{Gu}$ (2006) and collisional data (above ionization) from Bautista et al. (2004). We have also merged the A-values. Those for the allowed transitions of the lowest 342 close-coupling levels are from Butler \& Badnell (2008), while the remainder are from the previous CHIANTI model.

\subsection{F-like ions}

Witthoeft et al. (2007) calculated, within the APAP network, ICFT $R$-matrix calculations for electron-impact excitation amongst $195 n=3$ close-coupling levels for all F-like ions. The data for Si VI, S VIII, Ar X, Ca XII, Fe XVIII, and Ni XX were already added in CHIANTI version 6 (Dere et al. 2009).

For version 8 we have added the data for $\mathrm{Na}$ III, $\mathrm{Mg}$ IV, and Al v. The data for these lower charge state ions were not originally included in CHIANTI because they are of more limited accuracy than the data for the other ions in the sequence. However, these data are still a significant improvement, considering that previous CHIANTI versions only had three levels, and the excitation data for $\mathrm{Na}$ III and $\mathrm{Al} \mathrm{V}$ were interpolated along the sequence.

For Ne II, we have included the collision strengths calculated by Griffin et al. (2001). As noted by Witthoeft et al. (2007), the Griffin et al. (2001) close-coupling expansion was smaller 
(138 levels), however the target was more accurate because pseudo-orbitals were used to improve the level energies. The A-values for $\mathrm{Ne}$ II for the dipole-allowed transitions have been taken from Griffin et al. (2001), while A-values for a few M1, M2, E2 forbidden transitions have been taken from an ab initio MCHF calculation by Tachiev \& Froese Fischer (2002), see the MCHF collection ${ }^{5}$ at NIST.

To reduce the size of the data files containing the radiative rates, we have retained for all the new datasets only transitions with an A-value greater than $10^{-5}$ the largest A-value (for each level). Observed energies have been taken from NIST (Kramida et al. 2013).

\subsection{Ne-like ions}

Liang \& Badnell (2010) calculated electron-impact excitation of all Ne-like ions from $\mathrm{Na}^{+}$to $\mathrm{Kr}^{26+}$ using the ICFT $R$-matrix approach, for a large target comprising of 209 levels, up to outershell promotions to $n=7$. These calculations represent a significant improvement over the few previous calculations along the sequence. In general, we have adopted the Liang \& Badnell (2010) energies, A-values and collision strengths. To reduce the size of the data files containing the radiative rates, we have retained for all the new datasets only transitions with an A-value greater than $10^{-5}$ the largest A-value (for each level). The experimental energies are from NIST (Kramida et al. 2013), with few exceptions, most of which are noted below.

We have added the following datasets, which represent new ions not previously present in the CHIANTI database: Na II, Mg III, AlIV, P VI, KX, Ti XIII, Cr XV, MnXVI, Co XVIII and Zn XXI.

We have also replaced the previous model ions for Si V and $\mathrm{S}$ VII. The previous Si V and S VII model ions only had 27 levels, the ground configuration $2 p^{6}$ and the $2 p^{5} 3 s, 2 p^{5} 3 p, 2 p^{5} 3 d$ levels. In the case of $\mathrm{Si} \mathrm{V}$, the previous collision strengths were calculated with the DW approximation. In the case of S VII, we have adopted for most of the energies the experimental values of Jupén \& Engström (2002), while some are from NIST (Kramida et al. 2013).

Finally, we have replaced the previous model ions for Ar IX, Ca XI, and Ni XIX. The previous CHIANTI models for these ions had collision strengths from Zhang et al. (1987), calculated using a Coulomb-Born-Exchange method for the 88 levels arising from $n=3,4$ configurations.

For Ca XI, we have assessed the Ragozin et al. (1988) experimental energies, but found various inconsistencies, so we have adopted the NIST (Kramida et al. 2013) values instead. The important Fe XVII data were released previously in CHIANTI version 7 (Landi et al. 2012).

As in the case of the Li-like ions mentioned above, some of the collision strengths calculated by Liang \& Badnell (2010) were affected by a minor error which only affected the hightemperature rates. We used the corrected data (as obtained from the authors) for Mg III, P VI, Mn XVI, Si V, S VII, Ar IX, and Ni XIX.

For the astrophysically important ions Mg III, Al IV, Si V, S VII, ArIX, Ca XI, and Ni XIX we have replaced the Liang \& Badnell (2010) A-values of the transitions among the $2 \mathrm{p}^{6}$ and $2 p^{5} 31$ configurations (lowest 27 levels) with the results of the MCDHF calculations obtained by Jönsson et al. (2014) with the GRASP2K code (Jönsson et al. 2007). For some ions, the ordering of the levels was different, as well as the LSJ designation

\footnotetext{
5 http://nlte.nist.gov/MCHF/
}

of the dominant term. For all the strongest transitions, we found excellent agreement (to within a few percent) between the Liang \& Badnell (2010) and Jönsson et al. (2014) A-values.

\subsection{Na-like ions}

Within the UK APAP network, Liang et al. (2009b) calculated outer-shell electron-impact excitation of all Na-like ions from $\mathrm{Mg}^{+}$to $\mathrm{Kr}^{25+}$ using the ICFT $R$-matrix approach. The target and close-coupling expansion included 18 LS terms of configurations up to $n=6$. Liang et al. (2009c) presented the inner-shell electron-impact excitation of all Na-like ions from $\mathrm{Mg}^{+}$to $\mathrm{Kr}^{25+}$ with the ICFT $R$-matrix method with both Auger and radiation damping included via the optical potential approach. We have included these datasets for the astrophysically-important ions. The radiative data are taken from the same sets of calculations. To reduce the size of the data files containing the radiative rates, we have retained for all the new datasets only transitions with an A-value greater than $10^{-5}$ the largest A-value (for each level).

We have calculated (using AUTOSTRUCTURE version 24.24) the autoionization rates for the levels above ionization, using the same set of configurations and the same scaling parameters as adopted by Liang et al. (2009c).

For Al III, Si IV, P v, S VI, Ar VIII, K IX, Ca X, Cr XIV, Mn XV, Fe XVI, CoXVII, Ni XVIII the new $R$-matrix results replace the DW collision strengths of Sampson et al. (1990), and the innershell transitions are a new addition.

For $\mathrm{Mg}$ II, the new data replace the $R$-matrix calculations of Sigut \& Pradhan (1995), which included $10 \mathrm{LS}$ target terms. The method of calculation for this older calculation is very similar to the Liang et al. (2009b), so the latter results should be more accurate, given the larger target employed. We note that the $\mathrm{Mg}$ II $\mathrm{h}$ and $\mathrm{k}$ lines have a particular relevance for the recent solar spectroscopic mission IRIS; however, their emissivities are only slightly changed (of the order of $10 \%$ ).

For Ti XII and Zn XX, the Liang et al. (2009b) data replace the DW collision strengths calculated by Sampson et al. (1990).

\subsection{Si-like ions}

\subsubsection{S III}

New collisional data for S III have been calculated with the $R$-matrix suite of codes by Hudson et al. (2012), and have been included. They replace the previous $R$-matrix calculations from Tayal \& Gupta (1999). We have retained the A-values for the allowed and forbidden transitions we had in the previous CHIANTI version, calculated by Froese Fischer et al. (2006) with the ab initio MHCF method, and the Tayal \& Gupta (1999) A-values for the decays from the $4 d$ levels. Observed energies have been taken from NIST (Kramida et al. 2013).

We have compared the intensities of the brightest lines with the new model ion, and found some small increases (of the order of $20 \%$ ) for some transitions, due to the extra resonances related to the larger target.

\subsubsection{FeXIII}

The previous Fe XIII model included electron impact excitation rates calculated by Storey \& Zeippen (2010) with the $R$-matrix method for a total of 114 fine-structure levels, as well as DW data for the higher levels, up to $n=5$.

The new model includes APAP data from a much larger $R$-matrix calculation which included a total of 749 levels up to 
$n=4$ (Del Zanna \& Storey 2012). As discussed in Del Zanna \& Storey (2012), cascading from $n=5,6$ levels has a small effect for the strongest lines, so it is left out in the present model, to reduce the size of the ion.

The intensities of the transitions from the $n=3$ levels (in the EUV) are in close agreement with those of the previous model. The new model provides, however, more accurate data for the soft X-ray lines (decays from the $n=4$ levels) as compared to the previous version 7.1, which had collision strengths calculated with the DW approximation (see Del Zanna \& Storey 2012 for details). Observed energies have been taken from the benchmark works of Del Zanna (2011) and Del Zanna (2012a), where several new identifications in the EUV and in the soft X-rays were provided. Empirically-adjusted theoretical wavelengths as obtained by Del Zanna \& Storey (2012) are adopted.

\subsubsection{NixV}

Ni XV produces strong decays from the $3 s \mathrm{~s}^{3}$ and $3 \mathrm{~s}^{2} 3 \mathrm{p} 3 \mathrm{~d}$ configurations in the EUV region of the spectrum, several of which are observed by Hinode EIS, and are useful for density and abundance diagnostics of solar active regions (Del Zanna 2013). It also produces soft X-ray transitions from the $n=4$ levels.

We have included the recent APAP $R$-matrix calculations from Del Zanna et al. (2014c). They were obtained with a large target (up to $n=4$ levels), similar to the one adopted for Fe XIII. The new data replace the DW calculations of Landi \& Bhatia (2012).

\subsection{P-like ions}

\subsubsection{SII}

We have replaced the previous model with new collision strengths from Tayal \& Zatsarinny (2010). The A-values for the E1 transitions are taken from the paper, while the A-values of the E2 and M1 transitions have been calculated by O. Zatsarinny (priv. comm.). Observed energies have been taken from NIST (Kramida et al. 2013).

\subsubsection{FeXII}

The previous Fe XII model included electron impact excitation rates calculated by Storey et al. (2005) with the $R$-matrix codes for the lowest 143 levels, and DW data for the higher levels up to $n=5$.

The new model includes APAP data from a much larger $R$-matrix calculation which included 912 levels up to $n=4$ (Del Zanna et al. 2012a). The combined effect of extra cascading and increased excitation resulted in significantly (and surprisingly) different level populations of the levels of the ground configuration, which affected not only the forbidden lines, but also the electron density measurements obtained with Hinode EIS. The new model provides densities about a factor of three lower then the Storey et al. (2005) model. The intensities of the decays from the $3 \mathrm{~s} 3 \mathrm{p}^{4}$, observed in the EUV, are also increased. The extra cascading due to the $n=5,6$ levels was not found to be significant for the strongest transitions (less than $10 \%$, see Del Zanna et al. 2012a) so is not included in the present model, to reduce the size of the ion.

Observed energies have been taken from Del Zanna \& Mason (2005) and from Del Zanna (2012a) for the soft $\mathrm{X}$-ray lines. where several new identifications were provided.
Empirically-adjusted theoretical wavelengths as obtained by Del Zanna et al. (2012a) are used.

\subsection{S-like ions}

\subsubsection{SI}

$\mathrm{S} \mathrm{I}$ is a new addition to the database. We have included effective collision strengths from Tayal (2004). gf and E1 A-values have been obtained from Zatsarinny \& Bartschat (2006). We have noticed significant discrepancies between the two datasets for the allowed transitions. We have included the A-values for the forbidden transitions as calculated with the ab initio MHCF method by Irimia and Froese Fischer (unpublished, 2003, but as available at the MHCF database $\left.{ }^{6}\right)$. Experimental energies have been taken from NIST (Kramida et al. 2013).

\subsubsection{FeXI}

The previous Fe XI model included APAP electron impact excitation rates calculated by Del Zanna et al. (2010) with the $R$-matrix codes for the lowest 365 levels, and DW data for higher levels up to $n=5$. The target adopted by Del Zanna et al. (2010) was an ad hoc one, chosen to provide accurate collision strengths for transitions to three $n=3, J=1$ levels, which produce some of the strongest EUV lines for this ion. These levels have a strong spin-orbit interaction, and the collision strengths to these levels are very sensitive to the target. The accuracy of the target was confirmed a posteriori with comparisons against observations (Del Zanna 2010).

The new model includes APAP data from a much larger $R$-matrix calculation, which included 996 levels up to $n=4$ (Del Zanna \& Storey 2013). This calculation significantly improved the atomic data for many lower (within the $n=3$ ) and higher $(n=4)$ levels. As in the Fe XII case, Del Zanna \& Storey (2013) found that a number of lower $n=3$ levels had enhanced populations, caused by increased cascading from higher levels whose collision strengths are enhanced compared to the previous calculation.

The larger target, however, did not produce accurate collision strengths for the three $n=3, J=1$ levels, for which we have retained the collision strengths calculated by Del Zanna et al. (2010).

The new larger calculations significantly affected the predicted intensities of several strong EUV lines, some of which are observed by Hinode EIS. Experimental energies have been taken from Del Zanna (2010) and from Del Zanna (2012a), where several new identifications for the soft X-ray lines were provided. Empirically-adjusted theoretical wavelengths as obtained by Del Zanna \& Storey (2013) are adopted.

\subsection{Cl-like ions}

\subsubsection{FeX}

The new model ion includes a large-scale APAP $R$-matrix calculation (Del Zanna et al. 2012b) including 552 levels up to $n=4$. It replaces a previous APAP $R$-matrix calculation (Del Zanna et al. 2004) which included 54 levels up to $n=3$, and a DW calculation up to $n=5$.

6 http://nlte.nist.gov/MCHF/view.html 
The new atomic calculations for the Fe X $3 s^{2} 3 p^{4} 4 s$ levels showed that DW calculations significantly underestimate the collision strengths, as discussed in Del Zanna et al. (2012b).

The new larger calculations also significantly affect the predicted intensities of some EUV lines, in particular the strong decays from the $3 \mathrm{~s}^{2} 3 \mathrm{p}^{4} 3 \mathrm{~d}$ configuration observed by Hinode EIS (the $257.26 \AA$ self-blend), and from the $3 \mathrm{~s} 3 \mathrm{p}^{6}{ }^{2} \mathrm{~S}_{1 / 2}^{\mathrm{e}}$ level. Also, the intensities of the visible forbidden lines are significantly changed, as shown in Del Zanna et al. (2014b).

Experimental energies have been taken from Del Zanna et al. (2004) and from Del Zanna (2012a) for the soft X-ray lines. Empirically-adjusted theoretical wavelengths as obtained by Del Zanna et al. (2012b) are adopted.

\subsection{Ar-like ions}

\subsubsection{FeIX}

We have included the recent APAP $R$-matrix calculations from Del Zanna et al. (2014b), which replace the earlier calculations by Storey et al. (2002). The new data were obtained with a large target, which included the main levels up to $n=5$. Fe IX produces strong lines in the Hinode EIS spectral range, from the $3 s^{2} 3 p^{4} 3 d^{2}$ and $3 s^{2} 3 p^{5} 4 p$ configurations. They can be used to measure electron temperatures (Young 2009; Del Zanna et al. 2014b). The intensities of these lines are only slightly different, with the exception of the $197.854 \AA$ line, for which significant changes are present, as discussed in Del Zanna et al. (2014b). The new calculations also improve the data for several soft X-ray lines, decays from the $n=4,5$ levels, for which the previous CHIANTI version had DW data from O'Dwyer et al. (2012). We have retained the collision strengths and A-values of O'Dwyer et al. (2012) for the $3 s^{2} 3 p^{5} 61(1=s, p, d, f, g)$ configurations, by merging the two datasets.

\subsubsection{NiXI}

We have included the recent APAP $R$-matrix calculations from Del Zanna et al. (2014a). The target included the main configurations up to $n=4$, and is a significant improvement over the earlier $R$-matrix (only three $n=3$ configurations, calculated by Aggarwal \& Keenan 2007) and DW calculations (Bhatia $\&$ Landi 2011) which were included in the previous CHIANTI version. The two Ni XI lines at 207.9 and $211.4 \AA$ are observed by Hinode EIS. They are analogues of the Fe IX 241.7, $244.9 \AA$ lines, which are an excellent density diagnostic. The experimental energies discussed by Del Zanna et al. (2014a) are adopted.

\subsection{K-like ions}

\subsubsection{Fe VIII}

The collision strengths for Fe VIII have been notoriously difficult to calculate accurately. The previous version if the CHIANTI database contained the Griffin et al. (2000) electron excitation rates, scaled according to the benchmark structure calculations described in Del Zanna (2009). The adjustment improved agreement with observations of the EUV lines but was clearly not satisfactory. A new scattering calculation was carried out by Tayal \& Zatsarinny (2011) and significantly improved the Griffin et al. (2000) data. However, as pointed out in Del Zanna \& Badnell (2014), the Tayal \& Zatsarinny (2011) calculation did not improve the rates for all the transitions, in particular for some currently observed by the Hinode EIS spectrometer. A new large-scale (up to $n=5,518$ levels) $R$-matrix ICFT calculation has recently been carried out by Del Zanna \& Badnell (2014), adopting a new method which employs semi-empirical corrections based on the term energy corrections. The new data were shown by Del Zanna \& Badnell (2014) to substantially improve agreement for the EUV lines, some of which are useful to measure electron temperatures (Del Zanna 2009). The Del Zanna \& Badnell (2014) rates are adopted here, together with the corresponding A-values. The DW collision strengths and A-values for the extra 18 levels $(n=6,7)$ that were already present in CHIANTI, and were calculated by O'Dwyer et al. (2012) have been retained for the present model ion. The observed energies have been taken from the Del Zanna (2009) assessment and from NIST.

\subsection{Sc-like sequence}

\subsubsection{Fe VI}

We have included the $R$-matrix calculations of Ballance \& Griffin (2008) for Fe VI. The target included 96 close-coupling levels arising from the $3 \mathrm{~d}^{3}, 3 \mathrm{~d}^{2} 4 \mathrm{~s}, 3 \mathrm{~d}^{2} 4 \mathrm{p}$, and $3 \mathrm{p}^{5} 3 \mathrm{~d}^{4}$ configurations. They replace the previous calculations by Chen $\&$ Pradhan (1999) which included 80 close-coupling levels from the $3 \mathrm{~d}^{3}$ and the $3 \mathrm{~d}^{2} 4 \mathrm{~s}, 3 \mathrm{~d}^{2} 4 \mathrm{p}$ configurations. The A-values for the allowed transitions are taken from Ballance \& Griffin (2008), while those for the forbidden transitions are retained from the previous CHIANTI model, which originated from Chen \& Pradhan (2000).

\subsection{Cr-like sequence}

\subsubsection{Fe III}

Fe III is a new addition the CHIANTI database. This is a relatively complex ion for which several calculations and observations exist. In terms of scattering calculations, we first considered the data of Bautista et al. (2010) for the lowest 34 fine-structure levels (mainly the $3 \mathrm{~d}^{6}$ ), and the Iron Project calculations of Zhang (1996), which included excitations to a selection of $3 d^{5} 4 s$ and $3 d^{5} 4 p$ levels (for a total of 219 levels). However, we have chosen to include the most recent largescale calculations of Badnell \& Ballance (2014), which included excitation to 322 close-coupling levels, calculated with the Breit-Pauli $R$-matrix suite of codes. The authors did several comparisons with the results of other calculations, finding overall agreement, but suggested that the Breit-Pauli calculations should be more accurate.

Experimental energies have been taken from NIST (Kramida et al. 2013) and from Ekberg (1993). The ordering of the levels follows Badnell \& Ballance (2014). The assignment of the experimental energies was straightforward for the lowest levels (which produce the strongest lines for this ion), however the assignment to the higher levels was in several cases uncertain. Some discrepancies between NIST and Ekberg (1993) designations were also found.

We have also included, for consistency, the corresponding Avalues, which we recalculated with the same target adopted by Badnell \& Ballance (2014) using AUTOSTRUCTURE and the experimental energies whenever available. We included multipole orders up to three (E2/M1+Breit-Pauli corrections and E3/M2). Several previous calculations of radiative data also exist (cf. Deb \& Hibbert (2009) for the $3 d^{6}$ levels, and Ekberg (1993) for 1908 transitions from the $3 d^{5} 4 s$ and $3 d^{5} 4 p$ levels), and some 
differences are found for the weaker transitions. Considering the forbidden transitions within the $3 \mathrm{~d}^{6}$ levels, there is good agreement between the A-values calculated with AUTOSTRUCTURE with those of Deb \& Hibbert (2009).

\section{Other improvements}

\subsection{DEM inversion software}

The CHIANTI_DEM code has been modified substantially. The input and output basic files are the same, as well as the format of how the line contribution functions can be stored. The core inversion program has however been substantially modified. The older $\chi^{2}$ minimization program has been replaced with a more robust $\chi^{2}$ minimization program written by $\mathrm{M}$. Weber for the inversion of the Hinode XRT data (see, e.g. Weber et al. 2004), part of the Solarsoft program XRT_DEM_ITERATIVE2.

As an option, the routine can also run DATA2DEM_REG, a routine written by I. Hannah (described in Hannah \& Kontar 2012) and/or the Markov-Chain Monte-Carlo algorithm MCMC_DEM, written by V. Kayshap and described in Kashyap \& Drake (1998). In all three cases, the CHIANTI_DEM code saves the inputs as IDL save files, so users can subsequently rerun the inversion codes independently of CHIANTI_DEM. Details of the various parameters and where the programs can be downloaded can be found in the CHIANTI user guide and the CHIANTI_DEM documentation on the CHIANTI website.

\subsection{Improvements to ChiantiPy}

Landi et al. (2012) provided the first published description of the ChiantiPy ${ }^{7}$ software. The goal of ChiantiPy is to provide the ability to access the CHIANTI database and to compute line and continuum spectra. ChiantiPy is programmed in the Python ${ }^{8}$ programming language. The object oriented features of the language provide a good match to the structure of the CHIANTI database which is organized around single ions. This is reflected in ChiantiPy was the basic object is provided by the ion class.

In addition to the ion class, two spectrum classes spectrum and mspectrum, are available for calculation synthetic spectra for a group of ions. The mspectrum class implements the Python multiprocessing module to allow multiple processors such as the available cores on a single cpu or across a network. Two new multi-ion class have been developed, ipymspectrum and bunch. The ipymspectrum is very similar to the mspectrum except that it allows multiprocessing inside an IPython ${ }^{9}$ qtconsole or a notebook. In the latter, the IPython session is displayed inside a web browser. The other new multi-ion class bunch simply calculates the intensities, as well as other properties, for a selected set of ions.

Several new methods have been developed, intensityList() and convolve(). The previous ion methods intensityRatio() and intensityRatioSave() as well as the new method intensityList() are all now inherited by the ion class and the multi-ion classes spectrum, mspectrum, ipymspectrum and bunch. Consequently, it is now possible to calculate line intensity ratios as a function of temperature and density for lines of different ions.

The multi-ion classes have been restructured so that one can specify to keep all of the ion instances for later use. For example, one can calculated the spectrum selection of ions. This spectrum

\footnotetext{
7 http://sourceforge.net/projects/chiantipy

8 https://www.python.org/

9 http://ipython.org
}
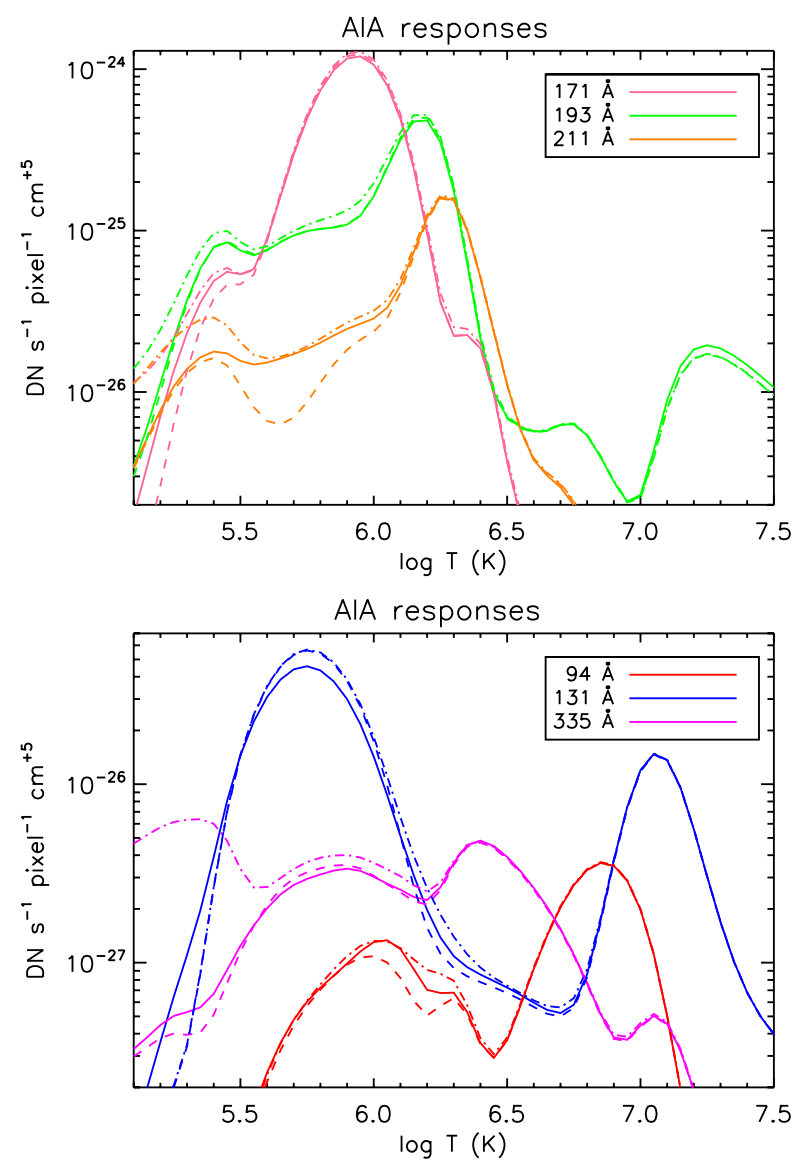

Fig. 1. SDO AIA responses. Full line: present data; dashed line: CHIANTI v.7.1.4; dot-dashed line: AIA software.

can then be compared with the spectrum of any of the ions in the initial selection. The convolve() methods can then perform a new convolution, with a different filter, of the ion intensities available in the ion instances. While useful, keeping all of the ion instances can be memory intensive. Consequently, the default option is not to keep the ion instances.

Another improvement is the ability to specify the elemental abundance directly to an ion instance and to specify the name of a file containing the elemental abundances directly to any of the multi-ion instances.

\subsection{AlA responses}

Figure 1 shows the SDO AIA responses calculated with the present atomic data, a constant pressure of $10^{15} \mathrm{~cm}^{-3} \mathrm{~K}$ and a set of "coronal" elemental abundances (Feldman 1992), where the elements with low First Ionization Potential such as iron are increased by about a factor of four, compared to their photospheric abundances.

The new responses are shown together with those of the previous CHIANTI version 7.1.4, and those obtained from the AIA software, at the same pressure and with the same elemental abundances. We can see that the most significant changes occur for the 131 and $94 \AA$ bands, for which the present data are a significant improvement.

Regarding the $131 \AA$ A band, we note that for low-temperature plasma or the quiet Sun the dominant emission lines are the Fe VIII 130.94 and 131.24 $\AA$ transitions (O'Dwyer et al. 2010; Del Zanna et al. 2011). The previous version of CHIANTI had 
atomic data for this ion that were semi-empirically adjusted by Del Zanna (2009). We note, however, that the adjustment was focused on improving the collision strengths for the EUV Fe VIII lines, and not those populating the $3 \mathrm{~s}^{2} 3 \mathrm{p}^{6} 4 \mathrm{f}^{2} \mathrm{~F}_{5 / 2,7 / 2}$ levels, which give rise to the 130.94 and $131.24 \AA$ lines, respectively.

The present atomic data also represent an improvement for the SDO AIA $94 \AA$ band. The previous version 7.1 of CHIANTI included DW collision strengths for Fe X. The Fe X $94.012 \AA$ transition was underestimated by at least $30 \%$, as shown in (Del Zanna et al. 2012b). Also, as pointed out in Del Zanna (2013), the intensity of the Fe $3 \mathrm{~s}^{2} 3 \mathrm{p}^{5}{ }^{2} \mathrm{P}_{3 / 2}-3 \mathrm{p}^{6} 3 \mathrm{~d}^{2} \mathrm{D}_{5 / 2}$ line at $94.27 \AA$ was incorrect. However, we recall that, as shown in Del Zanna (2013), a more significant contribution to the band in active region cores comes from an Fe XIV 93.61 A line, identified in Del Zanna (2012a). All the transitions observed within this band are now accounted for and have accurate atomic data, with the exception of a weak unidentified coronal line.

Finally, we caution users against using AIA responses for the 94 and $131 \AA$ channel that are empirically adjusted, as done for example with the /chiantifix keyword, as described in Boerner et al. (2014). These empirical adjustments do not have any justification when one considers in detail the underlying atomic data. For example, these adjustments modify the high-temperature peaks of the two bands, which are dominated by well-known lines from Fe XVIII and Fe XXI (O'Dwyer et al. 2010; Petkaki et al. 2012), for which the atomic data are accurate.

\section{Conclusions}

The present version 8 of CHIANTI represents a significant step towards one of our goals, to provide a consistent set of accurate atomic data for all the astrophysically important ions. Further work will focus on the ions in the Mg-like and Belike sequences, for which effective collision strengths have recently been calculated with the $R$-matrix ICFT method by the UK APAP network (Fernández-Menchero et al. 2014b,a). Inclusion of these data into CHIANTI has been delayed because Aggarwal \& Keenan (2015) have recently strongly questioned the accuracy of the $R$-matrix ICFT approach for the Be-like ions. With a detailed comparison, Fernández-Menchero et al. (2015) have however shown that the ICFT method produces the same results obtained with a fully relativistic Breit-Pauli $R$-matrix approach, which is needed for elements heavier than $\mathrm{Zn}$. The results obtained by Aggarwal and Keenan were actually more inaccurate because of their smaller configuration-interaction/close-coupling expansion.

We note that several other papers by Aggarwal and Keenan have also questioned the accuracy of the $R$-matrix ICFT approach for other ions, but the UK APAP data included in the present version of CHIANTI have been selected following a careful assessment against other data-sets to ensure their accuracy.

The present version also represent a significant improvement for the important but very complex coronal iron ions, which produce a very large number of spectral lines across the electromagnetic spectrum, and are used extensively for plasma diagnostic purposes.

The resonance structure near threshold often meant that $R$-matrix collision strengths could not be fitted accurately at low temperatures with a 5- or 9-point spline interpolation, so we have changed the format of the CHIANTI electron excitation files to store the data as they were calculated.
The current calculations of the line intensities are still carried out considering each ion separately, although ionization and recombination effects are taken into account with some approximations. The next version of CHIANTI will modify this approach.

Acknowledgements. A significant part of the present work was carried out by GDZ within SOLID, to improve atomic data for spectral irradiance modelling. SOLID (First European SOLar Irradiance Data Exploitation) is a collaborative SPACE Project under the Seventh Framework Programme (FP7/2007-2013) of the European Commission under Grant Agreement No. 313188. G.D.Z. and H.E.M. acknowledge support by the UK STFC bridging grant of the DAMTP astrophysics group at the University of Cambridge. P.R.Y. acknowledges support from NASA grant NNX15AF25G and NSF grant AGS-1159353. The work of E.L. was supported by NASA grant NNX11AC20G and NSF grant AGS1154443. The UK APAP network work was funded by STFC via the University of Strathclyde (grant No. PP/E001254/1 and ST/J000892/1). We thank all the colleagues that have provided us with electronic material. In particular we thank Oleg Zatsarinny, Manuel Bautista, Cathy Ramsbottom, Nigel Badnell, Guyiun Liang, Martin O'Mullane. We also thank Guyiun Liang for recalculating some of the atomic data and Nigel Badnell for advice on running AUTOSTRUCTURE.

\section{Appendix A: New file formats}

\section{A.1. File format definitions for the ELVLC files}

The format for the elvlc file remained the same from CHIANTI 1 through to CHIANTI 7.1. We have changed the format for version 8 , principally to allow level indices to go beyond 999, and remove duplicate information. Only fine structure levels are considered in CHIANTI, and for each level the configuration, L, S and J-values are given to describe the level. Energies are given in units of $\mathrm{cm}^{-1}$ and both an observed energy and a theoretical energy are given. If an observed energy does not exist, then the CHIANTI software uses the theoretical energy for that level. If an observed energy is not available for a level, then a "missing value" of -1 is assigned to that level. The CHIANTI theoretical energies are not necessarily the same as those of the scattering calculations. In some cases, theoretical "best guess" energies are provided. These are normally obtained by linear interpolation of the ab initio level energies with the few experimental energies. The wavelength file has the corresponding wavelengths. Typical uncertainties of the $a b$ initio wavelengths are a up to several $\AA$, while those obtained from the "best guess" energies are estimated to be around $1 \AA$.

The energy file contains columns with a fixed number of characters, and the data entries are terminated by a line containing only a -1 . All subsequent lines are considered to be comments.

There are eight data columns and each is described below. The format for the column is indicated by Fortran-style notation in Table A.1. Additional columns can be present in the files. These are for information purposes and are not read by the CHIANTI software.

Levels can be arranged in any order, although following the observed or theoretical energy ordering is recommended. Level 1 is recommended to be the ground level, although this is not essential. The usual configuration format is, e.g., $3 \mathrm{~s} 2.3 \mathrm{p} 2(2 \mathrm{P}) .3 \mathrm{~d}$. That is, orbitals are separated by a. and parent terms are placed in brackets. However, separating orbitals by white space is also present, e.g., $3 \mathrm{~s} 23 \mathrm{p} 2(2 \mathrm{P}) 3 \mathrm{~d}$.

We have added a level label string, which can be used to attach a label to a level. An example is for Fe II for which the strings are used for multiplets in the same configuration which have the same LSJ labels.

The elvlc file is read by the routine read_elvlc.pro, which has been modified to take into account the new format. In order 
Table A.1. Format of the data in the energy files.

\begin{tabular}{llll}
\hline \hline Col. & Format & ID & Comment \\
\hline 1 & i7 & LVL & Level index \\
2 & a30 & CONF & Configuration description \\
3 & a5 & LABEL & Level label string \\
4 & i5 & 2S+1 & Spin multiplicity \\
5 & a5 & L & Orbital angular momentum \\
6 & f5.1 & J & Total angular momentum \\
7 & f15.3 & E_o & Observed energy $\left(\mathrm{cm}^{-1}\right)$ \\
8 & f15.3 & E_b & "Best-guess" theoretical energy $\left(\mathrm{cm}^{-1}\right)$ \\
\hline
\end{tabular}

to maintain compatibility with the previous version of the routine, read_elvlc can be called in the identical manner to the old routine, i.e.,

IDL $>$ read_elvlc, filename, 11 , term, \$ conf , ss , $11, j \mathrm{j}$, ecm, eryd, ecmth, erydth, ref

However, read_elvlc can also be called with:

IDL> read_elvlc, filename, elvlcstr=elvlstr

where elvlcstr is an IDL structure containing the data. Note that elvlcstr has the tag elvlcstr.data.energy which contains the best guess energy for a level.

\section{A.2. File format definitions for the SCUPS files}

The SCUPS files replace the SPLUPS files in previous versions of the database. The format is very similar. For the new additions to version 8 , this file contains the temperatures and effective collision strengths, scaled using the Burgess \& Tully (1992) [BT92] method. The values as originally calculated can be obtained by descaling the data in the files, using the IDL routine DESCALE_ALL. In addition, the collision strengths at scaled temperatures of 0 and 1 are given. The value at threshold is extrapolated, while the value at scaled temperature equal to 1 is either extrapolated or obtained from the high-energy limit.

For the other ions that have not been modified in version 8 , the previous spline fits to the scaled effective collision strengths are retained. Only the format of the files is changed.

For each transition, there are three lines in the file. The first line in the SCUPS files contains the information about the transition, the second the BT92-scaled temperatures, and the third the BT92-scaled effective collision strengths, as described in Table A.2. Note that even in the cases when a single temperature array was present, with the new format each transition will have a different scaled temperature array.

The comments in the file are at the end, bracketed by two lines containing only a -1 .

The SCUPS file is read into an IDL structure as follows:

IDL> read_scups, splfile, splstr

where SPLSTR has two tags called INFO and DATA that are both structures. The tags for SPLSTR.INFO are listed in Table A.3.

The tags for SPLSTR.DATA are listed in Table A.4. The size of the TEMP and UPS arrays will be set to the maximum number of temperatures (NT_MAX) in the dataset. If e.g. NT_MAX=20 and a particular transition only has upsilons defined for 10 temperatures, then TEMP[0:9] and UPS[0:9] will contain these values, and TEMP[10:19] and UPS[10:19] will be set to the missing value, defined in UPSSTR.INFO.MISSING.
Table A.2. Format of the data in the SCUPS files.

\begin{tabular}{llll}
\hline \hline \multicolumn{2}{l}{ Col. Format } & ID & Comment \\
\hline 1 & i7 & L1 & Lower level of transition (integer) \\
2 & i7 & L2 & Upper level of transition (integer) \\
3 & e12.3 & DE & Energy of transition, Rydberg (float) \\
4 & e12.3 & GF & Oscillator strength (float) \\
5 & e12.3 & LIM & High-temperature limit value (float) \\
6 & i5 & NT & Number of scaled temperatures \\
7 & i3 & T_TYPE & BT92 Transition type (integer) \\
8 & e12.3 & C_VAL & BT92 scaling parameter (float) \\
\hline & e12.3 & SCT & Scaled temperatures (2nd line) \\
\hline & e12.3 & SCUPS & Scaled effective collision strengths (3rd line) \\
\hline
\end{tabular}

Table A.3. Tags for SPLSTR.INFO.

\begin{tabular}{lll}
\hline \hline Tag & Data & Type \\
\hline ION_NAME & Ion name (CHIANTI format) & String*1 \\
ION_Z & Atomic number & Integer*1 \\
ION_N & Spectroscopic number & Integer*1 \\
ION_ROMAN & Ion name (roman numerals) & String*1 \\
ION_LATEX & Ion name (latex format) & String*1 \\
ION_LATEX_ALT & Ion name (alternative latex format) & String*1 \\
COMMENTS & File comments & String array \\
CHIANTI_VER & version number & String*1 \\
TIME_STAMP & Time file was made & String*1 \\
FILENAME & Filename (including path) & String*1 \\
MISSING & Value for missing data & Float*1 \\
NTRANS & Number of transitions & Long*1 \\
\hline
\end{tabular}

Table A.4. Tags for SPLSTR.DATA.

\begin{tabular}{lll}
\hline \hline Tag & Data & Type \\
\hline LVL1 & Lower level index & Integer*1 \\
LVL2 & Upper level index & Integer*1 \\
DE & Energy (Rydberg) & Float*1 \\
GF & Oscillator strength & Float*1 \\
LIM & High-temperature limit & Float*1 \\
T_TYPE & BT92 Transition & Integer*1 \\
C_UPS & BT92 Scaling parameter & Float*1 \\
NSPL & Number of temperatures & Integer*1 \\
STEMP & Scaled temperature values & Float*NT_MAX \\
SPL & Scaled effective collision strength values & Float*NT_MAX \\
\hline
\end{tabular}

\section{References}

Aggarwal, K. M., \& Keenan, F. P. 2007, A\&A, 475, 393

Aggarwal, K. M., \& Keenan, F. P. 2008, A\&A, 486, 1053

Aggarwal, K. M., \& Keenan, F. P. 2015, MNRAS, 447, 3849

Aggarwal, K. M., Keenan, F. P., \& Heeter, R. F. 2009, Phys. Scr., 80, 045301

Artemyev, A. N., Shabaev, V. M., Yerokhin, V. A., Plunien, G., \& Soff, G. 2005, Phys. Rev. A, 71, 062104

Badnell, N. R. 2006, ApJS, 167, 334

Badnell, N. R. 2011, Comp. Phys. Comm., 182, 1528

Badnell, N. R., \& Ballance, C. P. 2014, ApJ, 785, 99

Badnell, N. R., Griffin, D. C., \& Mitnik, D. M. 2001, J. Phys. B Atom. Mol. Phys., 34, 5071

Ballance, C. P., \& Griffin, D. C. 2008, J. Phys. B Atom. Mol. Phys., 41, 195205

Bautista, M. A., Mendoza, C., Kallman, T. R., \& Palmeri, P. 2004, A\&A, 418, 1171

Bautista, M. A., Ballance, C. P., \& Quinet, P. 2010, ApJ, 718, L189

Beiersdorfer, P., \& Lepson, J. K. 2012, ApJS, 201, 28

Beiersdorfer, P., Träbert, E., Lepson, J. K., Brickhouse, N. S., \& Golub, L. 2014, ApJ, 788, 25

Bhatia, A. K., \& Landi, E. 2011, Atom. Data Nucl. Data Tables, 97, 50

Boerner, P. F., Testa, P., Warren, H., Weber, M. A., \& Schrijver, C. J. 2014, Sol. Phys., 289, 2377 
Burgess, A., \& Tully, J. A. 1992, A\&A, 254, 436 Butler, K., \& Badnell, N. R. 2008, A\&A, 489, 1369 Chang, E. S., \& Geller, M. 1998, Phys. Scr., 58, 326

Chen, G. X., \& Pradhan, A. K. 1999, J. Phys. B Atom. Mol. Phys., 32, 1809 Chen, G. X., \& Pradhan, A. K. 2000, A\&AS, 147, 111

Corrégé, G., \& Hibbert, A. 2004, Atom. Data Nucl. Data Tables, 86, 19

Dammasch, I. E., Wilhelm, K., Curdt, W., \& Hassler, D. M. 1999, A\&A, 346, 285

De Pontieu, B., Title, A. M., Lemen, J. R., et al. 2014, Sol. Phys., 289, 2733

Deb, N. C., \& Hibbert, A. 2009, J. Phys. B Atom. Mol. Phys., 42, 065003

Del Zanna, G. 2006, A\&A, 447, 761

Del Zanna, G. 2009, A\&A, 508, 513

Del Zanna, G. 2010, A\&A, 514, A41

Del Zanna, G. 2011, A\&A, 533, A12

Del Zanna, G. 2012a, A\&A, 546, A97

Del Zanna, G. 2012b, A\&A, 537, A38

Del Zanna, G. 2013, A\&A, 558, A73

Del Zanna, G., \& Badnell, N. R. 2014, A\&A, 570, A56

Del Zanna, G., \& Storey, P. J. 2012, A\&A, 543, A144

Del Zanna, G., \& Storey, P. J. 2013, A\&A, 549, A42

Del Zanna, G., \& Mason, H. E. 2005, A\&A, 433, 731

Del Zanna, G., Berrington, K. A., \& Mason, H. E. 2004, A\&A, 422, 731

Del Zanna, G., O’Dwyer, B., \& Mason, H. E. 2011, A\&A, 535, A46

Del Zanna, G., Storey, P. J., \& Mason, H. E. 2010, A\&A, 514, A40

Del Zanna, G., Storey, P. J., Badnell, N. R., \& Mason, H. E. 2012a, A\&A, 543, A139

Del Zanna, G., Storey, P. J., Badnell, N. R., \& Mason, H. E. 2012b, A\&A, 541, A90

Del Zanna, G., Storey, P. J., \& Badnell, N. R. 2014a, A\&A, 566, A123

Del Zanna, G., Storey, P. J., Badnell, N. R., \& Mason, H. E. 2014b, A\&A, 565, A77

Del Zanna, G., Storey, P. J., \& Mason, H. E. 2014c, A\&A, 567, A18

Dere, K. P., Landi, E., Mason, H. E., Monsignori Fossi, B. C., \& Young, P. R. 1997, A\&AS, 125, 149

Dere, K. P., Landi, E., Young, P. R., \& Del Zanna, G. 2001, ApJS, 134, 331

Dere, K. P., Landi, E., Young, P. R., et al. 2009, A\&A, 498, 915

Dudík, J., Del Zanna, G., Dzifčáková, E., Mason, H. E., \& Golub, L. 2014, ApJ, 780, L12

Ekberg, J. O. 1993, A\&AS, 101, 1

Feldman, U. 1992, Phys. Scr., 46, 202

Fernández-Menchero, L., Del Zanna, G., \& Badnell, N. R. 2014a, A\&A, 566, A104

Fernández-Menchero, L., Del Zanna, G., \& Badnell, N. R. 2014b, A\&A, 572, A115

Fernández-Menchero, L., Del Zanna, G., \& Badnell, N. R. 2015, MNRAS, 450, 4174

Froese Fischer, C., Tachiev, G., \& Irimia, A. 2006, Atom. Data Nucl. Data Tables, 92, 607

Goett, S. J., \& Sampson, D. H. 1983, Atom. Data Nucl. Data Tables, 29, 535

Griffin, D. C., Pindzola, M. S., \& Badnell, N. R. 2000, A\&AS, 142, 317

Griffin, D. C., Mitnik, D. M., \& Badnell, N. R. 2001, J. Phys. B Atom. Mol. Phys., 34, 4401

Gu, M. F. 2003, ApJ, 582, 1241

Gu, M. F. 2008, Canadian J. Phys., 86, 675

Gu, M. F., Schmidt, M., Beiersdorfer, P., et al. 2005, ApJ, 627, 1066

Hannah, I. G., \& Kontar, E. P. 2012, A\&A, 539, A146

Hibbert, A. 1975, Comput. Phys. Comm., 9, 141

Hibbert, A., Biemont, E., Godefroid, M., \& Vaeck, N. 1993, A\&AS, 99, 179

Hudson, C. E., Ramsbottom, C. A., \& Scott, M. P. 2012, ApJ, 750, 65

Jönsson, P., He, X., Froese Fischer, C., \& Grant, I. P. 2007, Comput. Phys. Comm., 177, 597

Jönsson, P., Bengtsson, P., Ekman, J., et al. 2014, Atom. Data Nucl. Data Tables, 100,1

Jupén, C., \& Engström, L. 2002, Phys. Scr., 66, 140

Kashyap, V., \& Drake, J. J. 1998, ApJ, 503, 450

Kato, T., Safronova, U., Shlypteseva, A., et al. 1997, Atom. Data Nucl. Data Tables, 67, 225

Kelly, R. L. 1987, Atomic and ionic spectrum lines below 2000 angstroms. hydrogen through krypton (New York: AIP, American Chemical Society and the National Bureau of Standards)

Kimura, E., Nakazaki, S., Berrington, K. A., \& Norrington, P. H. 2000, J. Phys. B Atom. Mol. Phys., 33, 3449

Kramida, A., Yu. Ralchenko, Reader, J., \& NIST ASD Team 2013, NIST Atomic Spectra Database (ver. 5.1), available: http://physics.nist. gov/asd (2014, July 30), National Institute of Standards and Technology, Gaithersburg, MD
Landi, E., \& Bhatia, A. K. 2012, Atom. Data Nucl. Data Tables, 98, 862

Landi, E., \& Gu, M. F. 2006, ApJ, 640, 1171

Landi, E., Del Zanna, G., Young, P. R., Dere, K. P., \& Mason, H. E. 2012, ApJ, 744, 99

Landi, E., Young, P. R., Dere, K. P., Del Zanna, G., \& Mason, H. E. 2013, ApJ, 763,86

Lepson, J. K., Beiersdorfer, P., Behar, E., \& Kahn, S. M. 2005, ApJ, 625, 1045 Liang, G. Y., \& Badnell, N. R. 2010, A\&A, 518, A64

Liang, G. Y., \& Badnell, N. R. 2011, A\&A, 528, A69

Liang, G. Y., Whiteford, A. D., \& Badnell, N. R. 2009a, A\&A, 499, 943

Liang, G. Y., Whiteford, A. D., \& Badnell, N. R. 2009b, A\&A, 500, 1263

Liang, G. Y., Whiteford, A. D., \& Badnell, N. R. 2009c, J. Phys. B Atom. Mol Phys., 42, 225002

Liang, G. Y., Badnell, N. R., \& Zhao, G. 2012, A\&A, 547, A87

Martin, W. C., Zalubas, R., \& Musgrove, A. 1990, J. Phys. Chem. Ref. Data, 19, 821

Martin, I., Karwowski, J., Diercksen, G. H. F., \& Barrientos, C. 1993, A\&AS, 100,595

Mewe, R., \& Gronenschild, E. H. B. M. 1981, A\&AS, 45, 11

Mewe, R., Gronenschild, E. H. B. M., \& van den Oord, G. H. J. 1985, A\&AS, 62, 197

Mitnik, D. M., Griffin, D. C., \& Badnell, N. R. 2001, J. Phys. B Atom. Mol. Phys., 34, 4455

O’Dwyer, B., Del Zanna, G., Mason, H. E., Weber, M. A., \& Tripathi, D. 2010, A\&A, 521, A21

O’Dwyer, B., Del Zanna, G., Badnell, N. R., Mason, H. E., \& Storey, P. J. 2012, A\&A, 537, A22

Peter, H., \& Judge, P. G. 1999, ApJ, 522, 1148

Petkaki, P., Del Zanna, G., Mason, H. E., \& Bradshaw, S. 2012, A\&A, 547, A25

Porquet, D., \& Dubau, J. 2000, A\&AS, 143, 495

Ragozin, E. N., Churilov, S. S., Kononov, E. Y., Ryabtsev, A. N., \& Zayikin, Y. F. 1988, Phys. Scr., 37, 742

Rynkun, P., Jönsson, P., Gaigalas, G., \& Froese Fischer, C. 2012, Atom. Data Nucl. Data Tables, 98, 481

Sampson, D. H., Goett, S. J., \& Clark, R. E. H. 1983, Atom. Data Nucl. Data Tables, 29, 467

Sampson, D. H., Weaver, G. M., Goett, S. J., Zhang, H., \& Clark, R. E. H. 1986, Atom. Data Nucl. Data Tables, 35, 223

Sampson, D. H., Zhang, H. L., \& Fontes, C. J. 1990, Atom. Data Nucl. Data Tables, 44, 209

Schmidt, M., Beiersdorfer, P., Chen, H., et al. 2004, ApJ, 604, 562

Sigut, T. A. A., \& Pradhan, A. K. 1995, J. Phys. B Atom. Mol. Phys., 28, 4879

Stafford, R. P., Bell, K. L., \& Hibbert, A. 1994, MNRAS, 266, 715

Storey, P. J., \& Zeippen, C. J. 2010, A\&A, 511, A78

Storey, P. J., Zeippen, C. J., \& Le Dourneuf, M. 2002, A\&A, 394, 753

Storey, P. J., Del Zanna, G., Mason, H. E., \& Zeippen, C. 2005, A\&A, 433, 717

Tachiev, G. I., \& Froese Fischer, C. 2002, A\&A, 385, 716

Tayal, S. S. 2000, Atom. Data Nucl. Data Tables, 76, 191

Tayal, S. S. 2004, ApJS, 153, 581

Tayal, S. S. 2006, ApJS, 163, 207

Tayal, S. S. 2008, A\&A, 486, 629

Tayal, S. S., \& Gupta, G. P. 1999, ApJ, 526, 544

Tayal, S. S., \& Zatsarinny, O. 2010, ApJS, 188, 32

Tayal, S. S., \& Zatsarinny, O. 2011, ApJ, 743, 206

Vainshtein, L. A., \& Safronova, U. I. 1978, Atom. Data Nucl. Data Tables, 21, 49

Wang, Y., Zatsarinny, O., \& Bartschat, K. 2013, Phys. Rev. A, 87, 012704

Weber, M. A., Deluca, E. E., Golub, L., \& Sette, A. L. 2004, in Multi-Wavelength Investigations of Solar Activity, eds. A. V. Stepanov, E. E. Benevolenskaya, \& A. G. Kosovichev, IAU Symp., 223, 321

Whiteford, A. D., Badnell, N. R., Ballance, C. P., et al. 2001, J. Phys. B Atom. Mol. Phys., 34, 3179

Witthoeft, M. C., Whiteford, A. D., \& Badnell, N. R. 2007, J. Phys. B Atom. Mol. Phys., 40, 2969

Young, P. R. 2009, ApJ, 691, L77

Young, P. R., Del Zanna, G., Landi, E., et al. 2003, ApJS, 144, 135

Zatsarinny, O., \& Bartschat, K. 2006, J. Phys. B Atom. Mol. Phys., 39, 2861

Zhang, H. 1996, A\&AS, 119, 523

Zhang, H., \& Sampson, D. H. 1987, ApJS, 63, 487

Zhang, H. L., Graziani, M., \& Pradhan, A. K. 1994, A\&A, 283, 319

Zhang, H., Sampson, D. H., Clark, R. E. H., \& Mann, J. B. 1987, Atom. Data Nucl. Data Tables, 37, 17

Zhang, H. L., \& Sampson, D. H. 1994, Atom. Data Nucl. Data Tables, 56, 41

Zhang, H. L., Sampson, D. H., \& Fontes, C. J. 1990, Atom. Data Nucl. Data Tables, 44, 31 